PROCEEDINGS OF THE

AMERICAN MATHEMATICAL SOCIETY

Volume 126, Number 1, January 1998, Pages 311-317

S $0002-9939(98) 04090-8$

\title{
CLOSURE ORDERING AND THE KOSTANT-SEKIGUCHI CORRESPONDENCE
}

\author{
DAN BARBASCH AND MARK R. SEPANSKI
}

(Communicated by Roe Goodman)

\begin{abstract}
Let $S$ be a real semisimple Lie group with Lie algebra $\mathfrak{s}=\mathfrak{k}+$ $\mathfrak{p}$. The Kostant-Sekiguchi correspondence is a bijection between nilpotent $S$ orbits on $\mathfrak{s}$ and nilpotent $K_{\mathbb{C}}$ orbits on $\mathfrak{p}_{\mathbb{C}}$. In this note we prove that the closure relations among nilpotent orbits are preserved under the KostantSekiguchi correspondence. The techniques rely on work of M. Vergne and P. Kronheimer.
\end{abstract}

\section{INTRODUCTION}

In its most general form, the Kostant-Sekiguchi correspondence establishes a bijection between nilpotent orbits on dual affine symmetric spaces. An important special case is the bijection between the nilpotent orbits of a real semisimple Lie group $S$ on its Lie algebra $\mathfrak{s}$, and the nilpotent orbits of the complexification of the maximal compact subgroup $K_{\mathbb{C}}$ on $\mathfrak{p}_{\mathbb{C}}$.

In [6] M. Vergne shows that this bijection in fact extends to a diffeomorphism between the orbits. The diffeomorphism uses results of P. Kronheimer in [3] in an essential way.

In this paper we remark that the Kostant-Sekiguchi correspondence in [5] also preserves closure relations. The necessary results are already in [6] where M. Vergne uses a special form of a correspondence in [3] between a set of flows and the nilpotent orbit. We simply substitute the more general correspondence in the case of a pair of orbits.

The results in this note imply that the closure relations of $K_{\mathbb{C}}$ orbits on $\mathfrak{p}_{\mathbb{C}}$ are determined by the closure relations of $S$ orbits on $\mathfrak{s}$ (notation as in section 2 ). The study of closure relations in the real and complex semisimple algebras has a long history. We refer to [4] for references on this subject.

\section{KRONHEIMER's RESULT}

In this section, we recall the necessary notation and theorems to make use of Kronheimer's model. For details, the reader should consult [3], [2], and [1].

Let $G$ be a compact, connected, semisimple Lie group with Lie algebra $\mathfrak{g}=$ $\operatorname{Lie}(G)$ and complexification $G_{\mathbb{C}}$ and $\mathfrak{g}_{\mathbb{C}}$, respectively. Kronheimer considers the

Received by the editors July 18, 1996.

1991 Mathematics Subject Classification. Primary 22E15, $17 \mathrm{~B} 05$.

Key words and phrases. Konstant-Sekiguchi, nilpotent orbits.

(C)1998 American Mathematical Society 
following trajectories which are the gradient flow of a certain function $f: \mathfrak{g} \times \mathfrak{g} \times \mathfrak{g} \rightarrow$ $\mathbb{R}$.

Definition 1.1. Write $\mathcal{M}$ for the set of all triples $A(t)=\left(A_{1}(t), A_{2}(t), A_{3}(t)\right)$ with $A_{j} \in \mathcal{C}^{\infty}(\mathbb{R}, \mathfrak{g})$ for $j \in\{1,2,3\}$ satisfying the three differential equations

$$
\begin{aligned}
& \frac{d}{d t} A_{1}=-2 A_{1}-\left[A_{2}, A_{3}\right], \\
& \frac{d}{d t} A_{2}=-2 A_{2}-\left[A_{3}, A_{1}\right], \\
& \frac{d}{d t} A_{3}=-2 A_{3}-\left[A_{1}, A_{2}\right] .
\end{aligned}
$$

To impose boundary conditions on the trajectories in $\mathcal{M}$, fix a basis $e_{1}, e_{2}, e_{3}$ for $\mathfrak{s u}(2)$ satisfying $\left[e_{1}, e_{2}\right]=-2 e_{3},\left[e_{2}, e_{3}\right]=-2 e_{1},\left[e_{3}, e_{1}\right]=-2 e_{2}$.

Definition 1.2. Given $\rho_{-}, \rho_{+}$homomorphisms from $\mathfrak{s u}(2)$ to $\mathfrak{g}$, write $\mathcal{M}\left(\rho_{-}, \rho_{+}\right)$ for the set of all $A \in \mathcal{M}$ satisfying

$$
\begin{gathered}
\lim _{t \rightarrow-\infty} A_{j}(t)=\left(g \rho_{-}\right)\left(e_{j}\right), \\
\lim _{t \rightarrow+\infty} A_{j}(t)=\rho_{+}\left(e_{j}\right)
\end{gathered}
$$

for some $g \in G$ and for all $j \in\{1,2,3\}$ where $g \rho_{-}$is the adjoint action on the homomorphism.

To relate $\mathcal{M}\left(\rho_{-}, \rho_{+}\right)$to the nilpotent variety, first fix a basis for $\mathfrak{s l}(2, \mathbb{C})$ by setting $H_{1}=i e_{1}, X_{1}=\frac{1}{2}\left(e_{2}+i e_{3}\right), Y_{1}=\frac{1}{2}\left(-e_{2}+i e_{3}\right)$. Cyclically permute $e_{1}, e_{2}, e_{3}$ to similarly define $H_{2}, X_{2}, Y_{2}$ and $H_{3}, X_{3}, Y_{3}$. Then for any homomorphism $\rho: \mathfrak{s u}(2) \rightarrow \mathfrak{g}$ and its complexification $\rho: \mathfrak{s l}(2, \mathbb{C}) \rightarrow \mathfrak{g}_{\mathbb{C}}$, write

$$
\mathcal{N}_{G_{\mathbb{C}}}(\rho)_{j}
$$

for the $G_{\mathbb{C}}$ orbit through $\rho Y_{j}$ in $\mathfrak{g}_{\mathbb{C}}$. Set

$$
S_{\mathfrak{g}_{\mathbb{C}}}(\rho)_{j}=\rho Y_{j}+\mathfrak{z}_{\mathfrak{g}_{\mathbb{C}}}\left(\rho X_{j}\right)
$$

where $\mathfrak{z}_{\mathfrak{g}}\left(\rho X_{j}\right)$ is the centralizer of $\rho X_{j}$ in $\mathfrak{g}_{\mathbb{C}}$.

Proposition 1.3. For $\rho, \rho^{\prime}$ homomorphisms from $\mathfrak{s u}(2)$ to $\mathfrak{g}$, the affine set $S_{\mathfrak{g}_{\mathbb{C}}}\left(\rho^{\prime}\right)_{j}$ is a transverse slice to the orbit $\mathcal{N}_{G_{\mathbb{C}}}\left(\rho^{\prime}\right)_{j}$. In particular, $\mathcal{N}_{G_{\mathbb{C}}}(\rho)_{j} \cap S_{\mathfrak{g}_{\mathbb{C}}}\left(\rho^{\prime}\right)_{j}$ is nonempty if and only if $\mathcal{N}_{G_{\mathbb{C}}}\left(\rho^{\prime}\right)_{j} \subseteq \overline{\mathcal{N}_{G_{\mathbb{C}}}(\rho)_{j}}$.

This is well known and follows from the representation theory of $\mathfrak{s l}(2)$. We omit the details.

Theorem 1.4 ([3], Theorem 1). For any pair of homomorphisms $\rho_{-}, \rho_{+}$, there is a natural diffeomorphism $R_{j}$ mapping

$$
\mathcal{M}\left(\rho_{-}, \rho_{+}\right) \cong \mathcal{N}_{G_{\mathbb{C}}}\left(\rho_{-}\right)_{j} \cap S_{\mathfrak{g}_{\mathbb{C}}}\left(\rho_{+}\right)_{j}
$$

for $j \in\{1,2,3\}$.

We review what we need from his proof. We set $j=1$ and suppress it from the notation.

First another Lie algebra valued function $A_{0}(t)$ is added so that $\left(A_{0}, A_{1}, A_{2}, A_{3}\right)$ satisfy the equations

$$
\frac{d}{d t} A_{1}=-2 A_{1}-\left[A_{0}, A_{1}\right]-\left[A_{2}, A_{3}\right]
$$




$$
\begin{aligned}
\frac{d}{d t} A_{2} & =-2 A_{2}-\left[A_{0}, A_{2}\right]-\left[A_{3}, A_{1}\right], \\
\frac{d}{d t} A_{3} & =-2 A_{3}-\left[A_{0}, A_{3}\right]-\left[A_{1}, A_{2}\right] .
\end{aligned}
$$

This new system is analyzed by considering the equivalent one in terms of the functions $\alpha=\frac{1}{2}\left(A_{0}+i A_{1}\right)$ and $\beta=\frac{1}{2}\left(A_{2}+i A_{3}\right)$ which take values in $\mathfrak{g}_{\mathbb{C}}$. These new functions satisfy the two equations

$$
\begin{gathered}
\frac{d}{d t}(\alpha+\bar{\alpha})+2(\alpha+\bar{\alpha})+2[\alpha, \bar{\alpha}]+2[\beta, \bar{\beta}]=0, \\
\frac{d}{d t} \beta+2 \beta+2[\alpha, \beta]=0 .
\end{gathered}
$$

The second equation is invariant under the complex gauge group $\mathcal{G}_{\mathbb{C}}$ of smooth maps

$$
g: \mathbb{R} \rightarrow G_{\mathbb{C}}, \quad(\alpha, \beta) \mapsto g \cdot(\alpha, \beta)=\left(\operatorname{Adg}(\alpha)-\frac{1}{2} \frac{d g}{d t} g^{-1}, A d g(\beta)\right) .
$$

This led Kronheimer to consider the following.

Definition 1.5. Given $\rho_{ \pm}$homomorphisms from $\mathfrak{s u}(2)$ to $\mathfrak{g}$, the complex trajectories associated to $\left(\rho_{+}, \rho_{-}\right)$are functions $(\alpha, \beta)$ satisfying

(i) equation (1.4) holds,

(ii) as $t \rightarrow+\infty$,

$$
\lim _{t \rightarrow+\infty} 2 \alpha(t)=H_{+}, \quad \lim _{t \rightarrow+\infty} \beta(t)=Y_{+},
$$

(iii) as $t \rightarrow-\infty$,

$$
\lim _{t \rightarrow-\infty} 2 \alpha(t)=A d g\left(H_{-}\right), \quad \lim _{t \rightarrow-\infty} \beta(t)=A d g\left(Y_{-}\right)
$$

for some $g$ in the compact group $G$,

(iv) $\alpha$ and $\beta$ approach their limits as $t \rightarrow \pm \infty$ with exponential decay, where $H_{ \pm}=\rho_{ \pm} H$ and $Y_{ \pm}=\rho_{ \pm} Y$.

Definition 1.6. Two complex trajectories $(\alpha, \beta)$ and $\left(\alpha^{\prime}, \beta^{\prime}\right)$ are called equivalent if there is a bounded gauge transformation $g \in \mathcal{G}_{\mathbb{C}}$ satisfying $g(t) \rightarrow 1$ as $t \rightarrow+\infty$ such that $\left(\alpha^{\prime}, \beta^{\prime}\right)=g \cdot(\alpha, \beta)$.

There are two main steps to the proof of Theorem 1.4. The first is:

Proposition 1.7 ([3], Section 4, Proposition 7, and the end of Sections 5 and 6). The equivalence classes of complex trajectories associated with $\left(\rho_{+}, \rho_{-}\right)$are parameterized by $\mathcal{N}_{G_{\mathbb{C}}}\left(\rho_{-}\right) \cap S_{\mathfrak{g}_{\mathbb{C}}}\left(\rho_{+}\right)$. Precisely, every complex trajectory is equivalent to a trajectory satisfying

$$
\begin{gathered}
(\alpha(t), \beta(t))=\left(\frac{1}{2} H_{-}, Y_{-}\right) \quad \text { for }-\infty<t \leq-1, \\
(\alpha(t), \beta(t))=\left(\frac{1}{2} H_{+}, Y_{+}+e^{-2 t} e^{-2 t a d H_{+}} \epsilon\right) \quad \text { for } 0 \leq t<\infty .
\end{gathered}
$$

The element $\epsilon$ can be chosen so that $Y_{+}+\epsilon \in S_{\mathfrak{g}_{\mathbb{C}}}\left(\rho_{+}\right)$in which case it is unique. Since $\beta(t) \in \mathcal{N}_{G_{\mathbb{C}}}\left(\rho_{-}\right)$for all $t, Y_{+}+\epsilon \in \mathcal{N}_{G_{\mathbb{C}}}\left(\rho_{-}\right) \cap S_{\mathfrak{g}_{\mathbb{C}}}\left(\rho_{+}\right)$. Conversely, given any $Y_{+}+\epsilon \in \mathcal{N}_{G_{\mathbb{C}}}\left(\rho_{-}\right) \cap S_{\mathfrak{g}_{\mathbb{C}}}\left(\rho_{+}\right)$, there exists a complex trajectory associated to $\left(\rho_{+}, \rho_{-}\right)$of the above form. 
The second step is:

Proposition 1.8 ([3], Section 4, Proposition 8). (a) For every complex trajectory $(\alpha, \beta)$, there is an equivalent trajectory $\left(\alpha^{\prime}, \beta^{\prime}\right)$ associated to $\left(\rho_{-}, \rho_{+}\right)$ that also satisfies Equation (1.3).

(b) If $(\alpha, \beta)$ and $\left(\alpha^{\prime}, \beta^{\prime}\right)$ are two equivalent complex trajectories associated to $\left(\rho_{-}, \rho_{+}\right)$both satisfying Equation (1.3) with $\alpha$ and $\alpha^{\prime}$ purely imaginary, then they are the same.

The outline of the proof of Theorem 1.4 is now possible. There is a well defined map from $\mathcal{M}\left(\rho_{-}, \rho_{+}\right)$to $\mathcal{N}_{G_{\mathbb{C}}}\left(\rho_{-}\right) \cap S_{\mathfrak{g}_{\mathbb{C}}}\left(\rho_{+}\right)$as follows: for $A \in \mathcal{M}\left(\rho_{-}, \rho_{+}\right)$, form a complex trajectory $(\alpha, \beta)$ by setting $A_{0}=0$ and use Proposition 1.7 to produce a unique element $Y_{+}+\epsilon \in \mathcal{N}_{G_{\mathbb{C}}}\left(\rho_{-}\right) \cap S_{\mathfrak{g}_{\mathbb{C}}}\left(\rho_{+}\right)$. The map is injective by the second part of Proposition 1.8. To see that the map is surjective, trace these steps backwards by starting with $Y_{+}+\epsilon \in \mathcal{N}_{G_{\mathbb{C}}}\left(\rho_{-}\right) \cap S_{\mathfrak{g}_{\mathbb{C}}}\left(\rho_{+}\right)$. Proposition 1.7 yields an appropriate complex trajectory $(\alpha, \beta)$ and the first part of Proposition 1.8 says that this trajectory is equivalent to one satisfying Equation (1.3) as well. Break this last trajectory into its real and imaginary parts to obtain $\left(A_{0}, A_{1}, A_{2}, A_{3}\right)$ satisfying Equation (1.2). Finally, apply a bounded gauge transformation $g \in \mathcal{G}_{\mathbb{C}}$ with values in $G$ and $\lim _{t \rightarrow+\infty} g(t)=1$ that transforms $A_{0}$ to 0 , i.e.,

$$
A d(g) A_{0}-\frac{d g}{d t} g^{-1}=0,
$$

to get the desired element in $\mathcal{M}\left(\rho_{-}, \rho_{+}\right)$.

\section{THE MAIN RESULT}

In this section we follow [6] very closely.

To get the three symmetric pairs used in the Sekiguchi correspondence, a certain quaternionic decomposition is needed. One way to get it is as follows.

Let $\mathfrak{s}$ be a real semi-simple Lie algebra with Cartan involution $\theta$ and corresponding Cartan decomposition $\mathfrak{s}=\mathfrak{k}+\mathfrak{p}$. Let $\sigma$ be another involution commuting with $\theta$ and write $\mathfrak{s}=\mathfrak{h}+\mathfrak{q}$ for the corresponding decomposition of $\mathfrak{s}$ with respect to the \pm 1 eigenvalues of $\sigma$. Set $\mathfrak{g}=\mathfrak{k}+i \mathfrak{p}$, a compact real form of $\mathfrak{s} \mathbb{C}$, and define

$$
\mathfrak{g}_{0}=\mathfrak{h} \cap \mathfrak{k}, \quad \mathfrak{g}_{1}=i(\mathfrak{h} \cap \mathfrak{p}), \quad \mathfrak{g}_{2}=\mathfrak{q} \cap \mathfrak{k}, \quad \mathfrak{g}_{3}=i(\mathfrak{q} \cap \mathfrak{p}) .
$$

This yields a quaternionic decomposition

$$
\mathfrak{g}=\mathfrak{g}_{0}+\mathfrak{g}_{1}+\mathfrak{g}_{2}+\mathfrak{g}_{3} .
$$

Let $G$ be a compact connected semisimple Lie group with Lie algebra $\operatorname{Lie}(G)=\mathfrak{g}$. Let $G^{0}$ the subgroup of $G$ with $\mathfrak{g}_{0}=\operatorname{Lie}\left(G^{0}\right), \mathfrak{h}^{j}=\mathfrak{g}_{0}+i \mathfrak{g}_{j}$ for $j \in\{1,2,3\}$, and $H^{j}$ the subgroup of $G_{\mathbb{C}}$ with $\mathfrak{h}^{j}=\operatorname{Lie}\left(H^{j}\right)$. Note that $G^{0}$ is the maximal compact subgroup of $H^{j}$. Finally, set

$$
\mathfrak{q}^{1}=\mathfrak{g}_{2}+i \mathfrak{g}_{3}, \quad \mathfrak{q}^{2}=\mathfrak{g}_{3}+i \mathfrak{g}_{1}, \quad \mathfrak{q}^{3}=\mathfrak{g}_{1}+i \mathfrak{g}_{2}
$$

which yields the three symmetric pairs $\left(\mathfrak{h}^{j}+\mathfrak{q}^{j}, \mathfrak{h}^{j}\right)$ used in the Sekiguchi correspondence in [5].

Definition 2.1. Given $\rho_{-}, \rho_{+}$homomorphisms from $\mathfrak{s u}(2)$ to $\mathfrak{g}$ with $\rho_{ \pm}\left(e_{j}\right) \in \mathfrak{g}_{j}$ for $j \in\{1,2,3\}$, write $\mathcal{S}\left(\rho_{-}, \rho_{+}\right)$for the set of all triples $A \in \mathcal{M}\left(\rho_{-}, \rho_{+}\right)$satisfying $A_{j}(t) \in \mathfrak{g}_{j}$. 
Definition 2.2. With $\rho_{-}, \rho_{+}$as above, define $\mathcal{S}_{0}\left(\rho_{-}, \rho_{+}\right)$to be all the triples $A \in$ $\mathcal{S}\left(\rho_{-}, \rho_{+}\right)$satisfying the stronger boundary condition at $-\infty$ of

$$
\lim _{t \rightarrow-\infty} A_{j}(t)=\left(g_{0} \rho_{-}\right)\left(e_{j}\right)
$$

for $j \in\{1,2,3\}$ and some $g_{0} \in G^{0}$.

The main result is:

Theorem 2.3. The restriction of $R_{j}$ to $\mathcal{S}_{0}\left(\rho_{-}, \rho_{+}\right)$from $\mathcal{M}\left(\rho_{-}, \rho_{+}\right)$yields a bijection

$$
\mathcal{S}_{0}\left(\rho_{-}, \rho_{+}\right) \cong \mathcal{N}_{H^{j}}\left(\rho_{-}\right)_{j} \cap S_{\mathfrak{q}^{j}}\left(\rho_{+}\right)_{j}
$$

for $j \in\{1,2,3\}$. Moreover, $R_{k} R_{j}^{-1}$ agrees with the Kostant-Sekiguchi correspondence on the orbit level.

As already mentioned, the proof is an elaboration of the case $\rho_{+}=0$ :

Theorem 2.4 ([6], Vergne). For $\rho_{-}$as above and $j \in\{1,2,3\}$, there is a diffeomorphism $R_{j}$ mapping

$$
\mathcal{S}_{0}\left(\rho_{-}, 0\right) \cong \mathcal{N}_{H^{j}}\left(\rho_{-}\right)_{j} .
$$

Moreover, $R_{k} R_{j}^{-1}$ commutes with the action of $G^{0}$, maps nilpotent $H^{j}$ orbits in $\mathfrak{q}^{j}$ to nilpotent $H^{k}$ orbits in $\mathfrak{q}^{k}$, and induces the Kostant-Sekiguchi correspondence.

We may now prove Theorem 2.3. Just as in [6], we consider the symmetric space $H^{j} / G^{0}$ instead of $G_{\mathbb{C}} / G$. The results in [3] Sections 5 and 6 hold without modification if we require complex trajectories to satisfy $(\alpha, \beta) \in\left(\mathfrak{h}^{j}, \mathfrak{q}^{j}\right)$ and use the appropriate gauge group $\mathcal{H}^{j}$ of smooth maps $h: \mathbb{R} \rightarrow H^{j}$.

Definition 2.5. Let $\rho_{ \pm}$be homomorphisms from $\mathfrak{s u}(2)$ to $\mathfrak{g}$ with $\rho_{ \pm}\left(e_{k}\right) \in \mathfrak{g}_{k}$ for $k \in\{1,2,3\}$. We say that $(\alpha, \beta)$ is an $\left(\mathfrak{h}^{j}, \mathfrak{q}^{j}\right)$ complex trajectory if $(\alpha, \beta)$ are functions taking values in $\left(\mathfrak{h}^{j}, \mathfrak{q}^{j}\right)$ and (i)-(iv) of Definition 1.5 hold with $G^{0}$ replacing $G$ in part (iii).

Two such complex trajectories are called equivalent if they are equivalent with respect to Definition 1.6 with $\mathcal{H}^{j}$ replacing $\mathcal{G}_{\mathbb{C}}$.

For the first part we set $j=1$. In this case, the results are:

Proposition 2.6. Suppose $(\alpha, \beta)$ is an $\left(\mathfrak{h}^{1}, \mathfrak{q}^{1}\right)$ complex trajectory. Then there is an equivalent trajectory, $\left(\alpha^{\prime}, \beta^{\prime}\right)$, satisfying:

$$
\begin{gathered}
\left(\alpha^{\prime}(t), \beta^{\prime}(t)\right)=\left(\frac{1}{2} H_{-}, Y_{-}\right) \quad \text { for }-\infty<t \leq-1, \\
\left(\alpha^{\prime}(t), \beta^{\prime}(t)\right)=\left(\frac{1}{2} H_{+}, Y_{+}+e^{-2 t} e^{-2 t a d H_{+}} \epsilon\right) \quad \text { for } 0 \leq t<\infty .
\end{gathered}
$$

The element $\epsilon$ can be chosen so that $Y_{+}+\epsilon \in S_{\mathfrak{q}^{1}}\left(\rho_{+}\right)_{1}$ and it is unique with this property. Furthermore $\beta(t) \in \mathcal{N}_{H^{1}}\left(\rho_{-}\right)_{1}$ for all $t$. Conversely, given any $Y_{+}+\epsilon \epsilon$ $\mathcal{N}_{H^{1}}\left(\rho_{-}\right)_{1} \cap S_{\mathfrak{q}^{1}}\left(\rho_{+}\right)_{1}$, there is an $\left(\mathfrak{h}^{1}, \mathfrak{q}^{1}\right)$ complex trajectory of the above form.

Proposition 2.7. Let $\rho_{ \pm}$be homomorphisms of $\mathfrak{s u}(2)$ to $\mathfrak{g}$ with $\rho_{ \pm}\left(e_{k}\right) \in \mathfrak{g}_{k}$.

(a) For every $\left(\mathfrak{h}^{j}, \mathfrak{q}^{j}\right)$ complex trajectory $(\alpha, \beta)$, there is an equivalent trajectory $\left(\alpha^{\prime}, \beta^{\prime}\right)$ associated to $\left(\rho_{-}, \rho_{+}\right)$that also satisfies Equation (1.3).

(b) If $(\alpha, \beta)$ and $\left(\alpha^{\prime}, \beta^{\prime}\right)$ are two equivalent $\left(\mathfrak{h}^{j}, \mathfrak{q}^{j}\right)$ complex trajectories associated to $\left(\rho_{-}, \rho_{+}\right)$both satisfying Equation (1.3) with $\alpha$ and $\alpha^{\prime}$ purely imaginary, then they are the same. 
Thus there is a well defined map, $R_{1}$, from $\mathcal{S}_{0}\left(\rho_{-}, \rho_{+}\right)$to $\mathcal{N}_{H^{1}}\left(\rho_{-}\right)_{1} \cap S_{\mathfrak{q}^{1}}\left(\rho_{+}\right)_{1}$ as follows. A solution to Equation (1.1) can be made into a solution to Equation (1.2) by setting $A_{0}=0$. Forming $(\alpha, \beta)$ as after Equation (1.2), the map is given by $(\alpha, \beta) \mapsto Y_{+}+\epsilon$ uniquely from Proposition 2.6. It is injective by the last part of Proposition 2.7. We need to show it is surjective. Let

$$
Y_{+}+\epsilon \in \mathcal{N}_{H^{1}}\left(\rho_{-}\right)_{1} \cap S_{\mathfrak{q}^{1}}\left(\rho_{+}\right)_{1} .
$$

Then let $(\alpha, \beta)$ be the corresponding trajectory as in Proposition 2.6. By the first part of Proposition 2.7, there is an $h \in \mathcal{H}^{1}$ so that $h \cdot(\alpha, \beta)$ satisfies Equation (1.3) as well. Writing this trajectory in the form $\frac{1}{2}\left(A_{0}+i A_{1}, A_{2}+i A_{3}\right)$ with $A_{i} \in \mathfrak{g}_{i}$, we obtain a solution to Equation (1.2). Finally, there exists $g \in \mathcal{H}^{1}$ with values in $G^{0}$ satisfying $\lim _{t \rightarrow+\infty} g(t)=1$ such that

$$
g(t) \cdot\left(A_{0}, A_{1}, A_{2}, A_{3}\right)=\left(0, A_{1}^{\prime}, A_{2}^{\prime}, A_{3}^{\prime}\right) .
$$

By construction, $\left(A_{1}^{\prime}, A_{2}^{\prime}, A_{3}^{\prime}\right)$ is the desired element.

We now complete the proof of the fact that the Kostant-Sekiguchi correspondence preserves the closure relations.

Lemma 2.8. The map

$$
\Psi: H^{j} \times S_{\mathfrak{q}^{j}}\left(\rho_{+}\right)_{j} \rightarrow \mathfrak{q}^{j}, \quad(h, X) \mapsto h X h^{-1}
$$

is a submersion. In particular $S_{\mathfrak{q}^{j}}\left(\rho_{+}\right)_{j}$ is a transverse slice to the $H^{j}$ orbits and $\mathcal{N}_{H^{j}}\left(\rho_{-}\right)_{j}$ contains $\mathcal{N}_{H^{j}}\left(\rho_{+}\right)_{j}$ in its closure if and only if $\mathcal{N}_{H^{j}}\left(\rho_{-}\right)_{j}$ intersects $S_{\mathfrak{q}^{j}}\left(\rho_{+}\right)_{j}$.

This is a slight generalization of Proposition 1.3 and we omit the proof as well.

Corollary 2.9. Let $i, j \in\{1,2,3\}$ be distinct. The $H^{i}$ orbit of $\rho_{-} Y_{i}$ contains $\rho_{+} Y_{i}$ in its closure if and only if the $H^{j}$ orbit of $\rho_{-} Y_{j}$ contains $\rho_{+} Y_{j}$ in its closure.

Proof. This follows from Theorem 2.3: the $H^{k}$ orbit of $\rho_{-} Y_{k}$ contains $\rho_{+} Y_{k}$ in its closure if and only if $\mathcal{N}_{H^{k}}\left(\rho_{-}\right)_{k} \cap S_{\mathfrak{q}^{k}}\left(\rho_{+}\right)_{k}$ is non-empty (Lemma 2.8) if and only if $\mathcal{S}_{0}\left(\rho_{-}, \rho_{+}\right)$is non-empty (the bijection $\left.R_{k}\right)$. This last condition is independent of whether $k=i$ or $k=j$.

Finally observe that since the Kostant-Sekiguchi correspondence maps $\mathcal{N}_{H^{i}}\left(\rho_{ \pm}\right)_{i}$ to $\mathcal{N}_{H^{j}}\left(\rho_{ \pm}\right)_{j}$, the proof of the remark in the Introduction is finished. To also see that $R_{j}^{-1} R_{i}$ agrees with the Kostant-Sekiguchi correspondence on the orbit level, note that $R_{j}^{-1} R_{i}$ maps $\rho_{+} Y_{i}+\epsilon_{i}$ to $\rho_{+} Y_{j}+\epsilon_{j}$ and that $\rho_{+} Y_{k}+\epsilon_{k}$ is $H^{k}$ conjugate to $\rho_{-} Y_{k}$.

\section{REFERENCES}

1. S. K. Donaldson. Nahm's equations and the classification of monopoles. Comm. Math. Phys., 96:387-407, 1984. MR 86c:58039

2. P. B. Kronheimer. A hyper-Kählerian structure on coadjoint orbits of a semisimple complex group. J. London Math. Soc., 42(2):193-208, 1990. MR 92b:53031

3. P. B. Kronheimer. Instantons and the geometry of the nilpotent variety. J. Differential Geometry, 32:473-490, 1990. MR 91m:58021

4. T. Ohta. The closures of nilpotent orbits in the classical symmetric pairs and their singularities. Tohoku Math. J., 43(2):161-211, 1991 MR 93c:22036 
5. J. Sekiguchi. Remarks on real nilpotent orbits of a symmetric pair. J. Math. Soc. Japan, 39:127-138, 1987. MR 88g:53053

6. M. Vergne. Instantons et correspondence de Kostant-Sekiguchi, C. R. Acad. Sci. Paris Sér. I Math. 320 (1995), 901-906. MR 96c:22026

Department of Mathematics, Cornell University, Ithaca, New York 14850

E-mail address: barbasch@math.cornell.edu

Department of Mathematics, Oklahoma State University, Stillwater, Oklahoma 74078

Current address: Department of Mathematics, Baylor University, Waco, Texas 76798-7328

E-mail address: Mark_Sepanski@Baylor.edu 